\title{
Significance of GH-IGF-I Axis in Changes in Body Composition, Bone and Lipid Metabolism in Perimenopausal Women
}

\author{
Kazuo Chihara and Toshitsugu Sugimoto
}

Third Division, Department of Medicine, Kobe University School of Medicine, Kobe, Japan

\begin{abstract}
Body composition as well as bone and lipid metabolism change during human aging irrespective of gender. In females, however, menopause is a critical event during aging which noticeably affects bone and lipid metabolism. The abrupt decrease in estrogen levels at menopause is supposed to reduce the tone of the GH-IGF-I axis although there is insufficient evidence to support this as yet. We investigated the association between the menopause and changes in the GH-IGF-I axis and body composition and tried to clarify the relationship between these parameters in perimenopausal women. The bone mineral density (BMD) of the lumbar spine and mid-radius of 77 healthy Japanese women aged 45-55 years (31 premenopausal [PRE] and 46 postmenopausal [POST]) was measured by dual energy X-ray absorptiometry (DEXA) and single photon absorptiometry, respectively. Body composition (bone mineral content [BMC], lean body mass [LBM] and fat mass) was also measured by DEXA and the percentage of BMC, LBM, and fat was calculated by dividing each body composition value by the total body mass. Percentage trunk fat was calculated by dividing the trunk fat by the total fat mass. The nocturnal urinary GH concentration normalized by the creatinine concentration was used as an index of endogenous GH secretion. Urinary GH and plasma IGF-I levels were significantly lower in the POST than in the PRE group, but plasma IGFBP-2 and -3 levels did not differ. The BMD of the lumber spine and mid-radius was significantly lower in the POST group and correlated positively with IGF-I but not IGFBP-2 or -3 levels; metabolic markers of bone formation and resorption were also significantly increased. \%BMC and \%trunk fat were significantly higher and lower, respectively, in the POST group than in the PRE group; serum total cholesterol and triglyceride level were also high in this group. Urinary GH levels correlated positively with \% LBM and negatively with \%fat, \%trunk fat, and serum total cholesterol and triglyceride levels; multiple regression analysis showed that this correlation was significant even when serum estradiol levels and age were taken into account. These parameters did not correlate with plasma IGF levels. These results indicate that menopause reduces the tone of the GH-IGF-I axis, which is probably responsible for decreased bone mass. Menopause also causes rapid changes in body composition and lipid metabolism, and these changes seem to correlate with the GH but not the IGF-I concentration.
\end{abstract}

Key words: GH, IGF-I, body composition, bone mineral density, menopause

Correspondence: Dr. Kazuo Chihara, Third Division, Department of Medicine, Kobe University School of Medicine, 7-5-1 Kusunoki-Cho, Chuo-Ku, Kobe 650, Japan 


\section{Introduction}

In a process of human aging, body composition as well as bone and lipid metabolism are markedly changed (1). GH secretion also decreases with age, which is accompanied by decreases in plasma insulin-like growth factor-I (IGF-I) and IGF-binding protein-3 (IGFBP-3) levels (2). The GH-IGF-I axis is known to regulate body composition (1). The menopause is a critical event during aging, which affects bone and lipid metabolism. An abrupt decrease in estrogen possibly reduces the tone of the GH-IGF-I axis, although there is insufficient evidence to support this as yet. It also remains unknown how the GHIGF-I axis is involved in changes in body composition as well as lipid metabolism during the perimenopausal period. On the other hand, the recent development of dual energy X-ray absorptiometry (DEXA) has made it possible to analyze body composition easily and precisely. Our previous study revealed that DEXA is useful for the analysis of age- and sex-dependent changes in body composition. The present study was performed to investigate how the menopause is associated with changes in the GH-IGF-I axis and body composition, bone and lipid metabolism, and to clarify the relationship between these parameters in perimenopausal women.

\section{Subjects and Methods}

Seventy-seven healthy Japanese women aged 45-55 years, who visited our out-patient clinic for bone mass measurement. They agreed to participate in this study and gave informed consent. None of the patients had liver or renal dysfunction, nutritional derangement or metabolic diseases which might cause changes in bone mass and/or body composition. All subjects were free of drugs known to influence bone and lipid metabolism, including estrogen replacement therapy.
They were divided into two groups according to their gonadal function. One group consisted of 31 premenopausal (PRE) women with menses. The other group was made up of 46 postmenopausal (POST) women who had been without spontaneous menses for at least one year before the beginning of the study. Serum estradiol levels in the POST group were all less than $20 \mathrm{pg} / \mathrm{ml}$.

\section{Measurements of bone mineral density (BMD) and body composition}

By means of DEXA (QDR-2000, Hologic Inc. Massachusetts) BMD of the lumbar spine L2-L4 was measured separately and expressed as the mean. Bone mineral content, bone width and BMD (defined as BMD/BW) were measured at the distal one-third of the radius by single photon absorptiometry (SPA) (Bone Mineral Analyzer Type 2780, Norland Co. WI). The same operator tested all the women during the study to eliminate operator discrepancies. The coefficients of variation (precision) of measurements of the lumbar spine and mid-radius were 0.9 and $1.9 \%$, respectively.

Body composition [bone mineral content (BMC), lean body mass (LBM) and fat mass] was also measured by DEXA with whole-body absorptiometry software, and each value was expressed in kilograms. The percentage of BMC, LBM and fat mass was calculated by dividing each absolute body composition value by the total body mass. The percentage of trunk fat was calculated by dividing the trunk fat by the total fat mass and was expressed as \% trunk fat. A strong correlation between body weight and total body mass measured by DEXA ( $r=0.98)$ was obtained in our preliminary study. The coefficients of variation (precision) of measurements of BMC, LBM and fat mass were $0.9,1.0$ and $2.0 \%$, respectively.

\section{Biochemical measurements}

There are several lines of evidence showing that the urinary $\mathrm{GH}$ level reflects the daily $\mathrm{GH}$ 
Table 1 BMD and biochemical data in perimenopausal women

\begin{tabular}{|c|c|c|c|c|c|}
\hline & & & Premenopausal & Postmenopausal & $p$ value \\
\hline \multicolumn{2}{|c|}{ Number of subjects } & & 31 & 46 & \\
\hline \multicolumn{2}{|l|}{ Age } & (Years) & $48.1 \pm 2.8$ & $52.8 \pm 2.6$ & $<0.01$ \\
\hline \multicolumn{2}{|c|}{ L2-4 BMD } & $\left(\mathrm{g} / \mathrm{cm}^{2}\right)$ & $0.932 \pm 0.111$ & $0.816 \pm 0.124$ & $<0.01$ \\
\hline \multicolumn{2}{|c|}{ Mid-radius BMD } & $\left(\mathrm{g} / \mathrm{cm}^{2}\right)$ & $0.642 \pm \quad 0.048$ & $0.597 \pm 0.086$ & $<0.01$ \\
\hline \multirow[t]{6}{*}{ Serum } & $\mathrm{Ca}$ & (mg/dl) & $9.01 \pm 0.40$ & $9.16 \pm 0.35$ & NS \\
\hline & $\mathrm{P}$ & (mg/dl) & $3.52 \pm 0.43$ & $3.62 \pm 0.49$ & NS \\
\hline & ALP & (IU/A) & $191.8 \pm 51.9$ & $241.2 \pm 65.1$ & $<0.01$ \\
\hline & Osteocalcin & (ng/ml) & $4.27 \pm 2.76$ & $7.48 \pm 3.63$ & $<0.01$ \\
\hline & Bone-ALP & $(\mathrm{U} / \mathrm{l})$ & $16.07 \pm 5.02$ & $24.50 \pm$ & $<0.01$ \\
\hline & TRAP & (IU/) & $4.60 \pm 0.85$ & $5.49 \pm$ & $<0.05$ \\
\hline \multirow[t]{3}{*}{ Urinary } & $\mathrm{Ca} /$ Creat & & $0.20 \pm$ & $0.27 \pm$ & $<0.05$ \\
\hline & Pyr/Creat & $(\mathrm{pmol} / \mu \mathrm{mol})$ & $25.78 \pm$ & $31.23 \pm$ & $<0.01$ \\
\hline & D-Pyr/Creat & $(\mathrm{pmol} / \mu \mathrm{mol})$ & $4.60 \pm 1.59$ & $6.66 \pm$ & $<0.01$ \\
\hline
\end{tabular}

secretion rate. Since the daily main GH peak in adults appears during the early nocturnal phase of the sleep period, we collected the urine just after waking up in the morning. The GH concentration divided by the creatinine concentration in urine samples was used as an index of endogenous GH secretion. The urinary GH level was measured by sandwich enzyme immunoassay.

After overnight fasting, plasma was collected, promptly separated and stored at $-20 \mathrm{C}$ until assayed. The plasma IGF-I level was measured by radioimmunoassay (RIA) after acid-ethanol extraction. Plasma IGFBP-2 and 3 levels were also measured by RIA. Intra- and interassay variations in these measurements were 3 to $4 \%$ and 7 to $8 \%$, respectively.

\section{Statistical analysis}

Data were expressed as the mean \pm SD. Statistical significance was determined by Student's t-test. P-values less than 0.05 were considered significant. The regression analysis was performed with the statistical computer program Abacus Concepts, Statview (Abavus concepts, Inc., Berkley, CA). Simple regression analysis was used to assess the linear relationship be- tween study parameters, and then Pearson's correlation coefficients were calculated. Multiple regression analyses were also employed.

\section{Results and Discussion}

Table 1 shows the background data of the subjects examined. The age of the POST group was significantly higher than that of the PRE group, but the difference was very small. BMD levels for the lumbar spine and mid-radius were significantly lower in the POST group. Serum total alkaline phosphatase (ALP) levels and the urinary calcium $(\mathrm{Ca})$ to creatinine $(\mathrm{Cr})$ ratio were significantly higher in the POST group. Moreover, the levels of metabolic markers of bone formation such as serum osteocalcin and boneALP levels as well as of bone resorption such as serum tartrate-resistant acid phosphatase (TRAP) levels and the ratio of urinary pyridinoline and deoxypyridinoline to $\mathrm{Cr}$ were all significantly higher in the POST group, indicating that the bone turnover rate is accelerated after menopause, thereby causing the loss of bone mass.

Urinary GH and plasma IGF-I levels were significantly lower in the POST group than in 
Table 2 Body composition and serum lipid data in perimenopausal women

\begin{tabular}{ccccl}
\hline & \multicolumn{5}{c}{ Premenopausal } & Postmenopausal & p value \\
\hline Body composition & & & & \\
BMC & $(\mathrm{Kg})$ & $1.784 \pm 0.196$ & $1.624 \pm 0.279$ & $<0.01$ \\
LBM & $(\mathrm{Kg})$ & $33.307 \pm 3.280$ & $32.024 \pm 3.312$ & $\mathrm{NS}$ \\
FAT & $(\mathrm{Kg})$ & $16.863 \pm 1.054$ & $17.885 \pm 4.915$ & $\mathrm{NS}$ \\
\%BMC & & $3.47 \pm 0.42$ & $3.18 \pm 0.48$ & $<0.01$ \\
\%LBM & & $64.77 \pm 7.13$ & $62.45 \pm 7.00$ & $\mathrm{NS}$ \\
\%FAT & & $31.76 \pm 7.37$ & $34.01 \pm 6.620$ & $\mathrm{NS}$ \\
BMC/LBM & & $0.054 \pm 0.006$ & $0.050 \pm 0.007$ & $<0.05$ \\
BMC/FAT & & $0.119 \pm 0.047$ & $0.099 \pm 0.037$ & $<0.05$ \\
FAT/LBM & & $0.508 \pm 0.166$ & $0.562 \pm 0.164$ & $\mathrm{NS}$ \\
\% Trunk FAT & & $38.30 \pm 7.83$ & $42.47 \pm 7.11$ & $<0.05$ \\
T-cholesterol & $(\mathrm{mg} / \mathrm{dl})$ & $194.5 \pm 26.9$ & $221.2 \pm 36.8$ & $<0.01$ \\
Triglyceride & (mg/dl) & $87.8 \pm 32.5$ & $119.2 \pm 69.9$ & $<0.05$ \\
\hline
\end{tabular}

the PRE group, although plasma IGFBP-2 and 3 levels were different. Circulating IGF-I is believed to be derived mainly from the liver and its level reflects the amount of $\mathrm{GH}$ secreted unless the subjects suffer from liver dysfunction, malnutrition, etc. (3). In addition, osteoblasts locally synthesize IGF-I and IGFBP-3 $(4,5)$, so bone might be a substantial source of plasma IGF-I and IGFBP-3. Besides, the GH secretion rate declines after menopause whereas estrogen exerts stimulatory actions on a number of sites in the GH/IGF-I axis. Indeed, estrogen stimulates IGF-I and IGFBP-3 production in osteoblasts. Taken together, the significant reduction in both urinary GH and plasma IGF-I in the POST group would be explained by an abrupt decrease in estrogen at menopause. Despite attenuation of the GH-IGF-I axis in the POST group, the plasma IGFBP-3 level did not change after menopause. But this is compatible with the recent report indicating that estrogen treatment does not change the plasma IGFBP-3 level in healthy postmenopausal women (6). The mechanisms of regulating IGFBP-3 production remain unclear. Blum et al. (7) reported that IGFBP-3 is primarily regulated by $\mathrm{GH}$, but Corpas et al. (8) reported that in elderly people the plasma
IGFBP-3 level depends on IGF-I but not on spontaneous GH release so that a regulator other than GH or IGF-I seems to be responsible for the determination of the serum IGFBP-3 level in the perimenopausal period.

A significant decrease in plasma IGF-I after menopause might be partly involved in the bone loss. It is of interest that plasma IGF-I levels were positively correlated with the BMD of the lumbar spine and mid-radius. Although local factors such as interleukin 1 and 6 are proposed to play a role in the pathogenesis of bone loss secondary to an abrupt decrease in estrogen (9, $10)$, the present finding suggests that IGF-I might also be partly involved in the pathogenesis of postmenopausal osteoporosis.

Table 2 shows the changes in body composition and serum lipid levels in perimenopausal women. In the POST group, \% BMC was significantly lower and \%trunk fat was significantly higher than in the PRE group, and \%LBM and $\%$ fat tended to be lower and higher in the POST group, respectively, although these were not statistically significant. Serum total cholesterol and triglyceride levels were quite high in the POST group. These data indicate that menopause causes rapid changes in body composition and lipid 
metabolism.

We analyzed the correlation of GH and IGFI levels in the changes in body composition during perimenopause. The urinary GH level was positively correlated with \%BMC and \%LBM, but negatively correlated with \% fat. Multiple regression analysis revealed that the positive correlation of $\mathrm{GH}$ with both \% BMC and \% LBM as well as the negative correlation of $\mathrm{GH}$ with \% fat were evident even if age and the plasma estradiol level were taken into account. It was to our surprise that there was no correlation between the plasma IGF-I level and body composition since IGF-I is believed to be a mediator of GH action in the metabolism of bone, muscle and fat, etc. The present findings suggest that $\mathrm{GH}$ itself plays an important role in the regulation of body composition during perimenopause mainly through an IGF-I-independent pathway.

The age-dependent decrease in GH secretion is reported to be strongly related to the proportion of abdominal body fat (11). GH administration to both GH-deficient children and adults prompted a redistribution of adipose tissue from the abdomen to the periphery (12)and decreased lipogenesis in abdominal fat tissue (13). In the present study, the urinary GH level, but not the plasma IGF-I level, was negatively correlated with \% trunk fat, and this relationship was also significant even if the factors of age and plasma estradiol were taken into account, suggesting that the abdominal fat increase caused by $\mathrm{GH}$ deficiency during perimenopause is not mediated by IGF-I.

Since serum total cholesterol and triglyceride levels were significantly increased in the POST group, we next analyzed the correlation of GH and IGF-I levels in the increase in serum total cholesterol and triglyceride. Serum total cholesterol and triglyceride levels were negatively correlated with the GH level, but not with plasma IGF-I. Multiple regression analysis revealed that negative relationships between $\mathrm{GH}$ and serum lipid levels were also significant even if age and the serum estradiol level were taken into account, suggesting that an decrease in the $\mathrm{GH}$ secretion rate after menopause, but not IGF-I, is at least in part linked to the changes in serum lipid levels during perimenopause.

Aging is associated with bone loss and body composition changes such as muscle mass decrease and fat mass increase. Our recent study revealed that an age-related decline in $\mathrm{GH}$ secretion was involved in age-dependent bone loss and changes in body composition in elderly women (14). The present study focusing on perimenopausal women indicates that $\mathrm{GH}$ itself plays a crucial role in the physiological regulation and maintenance of bone mass and body composition.

In conclusion, menopause caused decreased production of GH and IGF-I, both of which are probably responsible for decreased bone mass, whereas menopause led to rapid changes in body composition and lipid metabolism which can be mainly attributed to the reduced production of GH but not of IGF-I.

\section{References}

1. Rudman D. Growth hormone, body composition and aging. J Am Geriatr Soc 1985; 33: 800-7.

2. Corpas E, Harman SM, Blackman MR. Human growth hormone and human aging. Endocrine Rev 1993; 14: 20-39.

3. Schwander JC, Hauri C, Zapf J, Froesch ER. Synthesis and secretion of insulin-like growth factor and its binding protein by the perfused rat liver: dependence on growth hormone status. Endocrinology 1983; 113: 297-305.

4. Ernst M, Froesch ER. Growth hormone dependent stimulation of osteoblast-like cells in serum-free cultures via local synthesis of insulin-like growth factor I. Biochem. Biophys Res Commun 1988; 151: 142-7.

5. Schmid C, Schlapfer I, Peter M, Boni-Schnetzler M, Schwander J, Zapf J. Growth hormone and parathyroid hormone stimulate IGFBP-3 in rat osteoblasts. Am J Physiol 1994; 267: E226-33. 
6. Shewmon DA, Stock JL, Rosen CJ. Tamoxifen and estrogen lower circulating lipoprotein(a) concentrations in healthy postmenopausal women. Arterioscler Thromb 1994; 14: 1586-93.

7. Blum WF, Ranke MB. Insulin-like growth factor binding proteins (IGFBPs) with special reference to IGFBP-3. Acta Paediatr Scand 1990; 367: 5562.

8. Corpas E, Harman SM, Blackman MR. Serum IGFbinding protein-3 is released to IGF-I, but not to spontaneous $\mathrm{GH}$ release, in healthy old men. Horm Metab Res 1992; 24: 543-5.

9. Pacifici R, Rifas L, McCracken R. Ovarian steroid treatment blocks a postmenopausal increase in blood monocyte interleukin 1 release. Proc Natl Acad Sci USA 1989; 86: 2398-402.

10. Jilka RL, Hangoc G, Girasole G. Increased osteoclast development after estrogen loss: Mediation by interleukin 6 . Science 1992; 257: 88-91.

11. Boer HD, Blok G-J, Van der Leen EA. Clinical aspects of growth hormone deficiency in adults. Endocrine Rev 1995; 16: 63-86.

12. Parra A, Argote RM, Garcia G. Body composition in hypopituitary dwarfs before and during human growth hormone therapy. Metabolism 1979; 28: 851-9.

13. Tanner JM, Hughes PCR, Whitehouse RH. Comparative rapidity of response of height, limb muscle and limb fat to treatment with human growth hormone in patients with and without growth hormone deficiency. Acta Endocrinol 1977; 84: 681-96.

14. Nasu M, Sugimoto T, Chihara M, Hiraumi S, Kurimoto F, Chihara K. Effect of natural menopause on serum levels of insulin-like growth factor-I (IGF-I) and IGF binding proteins : relationship with bone mineral density and lipid metabolism in perimenopausal women. Eur $\mathrm{J}$ Endocrinol 1997 (In press). 